\title{
Comparative Characterisation of Genotypically Different Clones of MRSA in the Production of Biofilms
}

\author{
Salman Sahab Atshan, ${ }^{1,2}$ Mariana Nor Shamsudin,, ${ }^{1,3}$ Leslie Than Thian Lung, \\ Zamberi Sekawi, ${ }^{1}$ Ehsanollah Ghaznavi-Rad, ${ }^{4}$ and Chong Pei Pei ${ }^{5}$ \\ ${ }^{1}$ Department of Medical Microbiology and Parasitology, Faculty of Medicine and Health Science, \\ Universiti Putra Malaysia, Selangor, Serdang, Malaysia \\ ${ }^{2}$ Department of Medical Microbiology, Basrah University, Basrah, Iraq \\ ${ }^{3}$ Department of Marine Science and Aquaculture, Institute of Bioscience, Universiti Putra Malaysia, \\ Selangor, Serdang, Malaysia \\ ${ }^{4}$ Department of Microbiology and Immunology, Arak University of Medical Sciences, Arak, Iran \\ ${ }^{5}$ Department of Obstetrics and Gynaecology, Faculty of Medicine and Health Science, \\ Universiti Putra Malaysia, Selangor, Serdang, Malaysia
}

Correspondence should be addressed to Salman Sahab Atshan, salmanatshan@yahoo.com

Received 5 October 2011; Revised 13 December 2011; Accepted 14 December 2011

Academic Editor: Frederick D. Quinn

Copyright ( $) 2012$ Salman Sahab Atshan et al. This is an open access article distributed under the Creative Commons Attribution License, which permits unrestricted use, distribution, and reproduction in any medium, provided the original work is properly cited.

\begin{abstract}
The ability to adhere and produce biofilms is characteristic of enhanced virulence among isolates of methicillin-resistant Staphylococcus aureus (MRSA). The aim of the study is to find out whether these characteristics are consistently similar among isolates variations of MRSA. The study used 30 various isolates of MRSA belong to 13 spa types and 5 MLST types and determined the aggregation, the adherence, and the production of biofilms and slime for each isolate. The methods used to evaluate these characteristics were a modified Congo red agar assay (MCRA), a microtiter plate assay (MPA), high-magnification light microscopy, scanning electron microscopy (SEM), and PCR. The study found that isolates belonging to similar Spa, SCCmec, and ST types have similar abilities to produce biofilms; however, their ability to produce slime on CRA was found to be different. Moreover, isolates that have different Spa types showed high variation in their ability to produce biofilms. The results of light microscope revealed the isolates that produced strong and weak biofilms and formed similar aggregation on the glass surfaces. SEM results showed that all 30 MRSA isolates that were tested were $100 \%$ positive for biofilm formation, although to varying degrees. Further testing using PCR confirmed that $100 \%$ of the 30 isolates tested were positive for the presence of the icaADBC, $f n b A$, eno, ebps, clfA, and $c l f B$ genes. The prevalence of $f i b, c n a, f n b B$, and $b b p$ in MRSA clones was 90, 93.33, 53.33, and 10\%, respectively. This study indicate that differences in biofilm production capacities are caused by the differences in surface protein $A$ (Spa) type and are not due to differences in MLST and SCCmec types.
\end{abstract}

\section{Introduction}

Many bacterial pathogens and nosocomial infections are the cause of acute and chronic infections due to their ability to form biofilms $[1,2]$. Even though biofilm-forming properties have been well demonstrated by the members of the Staphylococcus genus such as S. epidermidis and S. aureus, it is less studied in modern methicillin-resistant Staphylococcus aureus (MRSA), which has evolved from several clonal lineages of methicillin-susceptible S. aureus strains via acquisition of a mobile genetic element called Staphylococcal cassette chromosome mec (SCCmec). The ability of MRSA to produce biofilm has resulted in difficultly in understanding its high clonal diversity, including its enhanced propensity to spread and cause opportunistic human infections in various parts of the world. The initial bacterial monolayer that sticks to a polymeric surface changes to a common biofilm that includes bacteria and an extracellular slime substance. The proliferation of the bacteria and the formation of the slime results in a higher resistance to antibiotics because drugs are 
prevented from reaching the bacteria that are protected by biofilm [3]. Many studies have concluded that the formation of the biofilm is caused by adherence at late stages of bacterial growth. In this process, the organisms stick to each other through polysaccharide intercellular adhesion (PIA), which is synthesised by products of the icaADBC operon [4]. Thus, it is important to study the ability of different MRSA clones to produce biofilms in order to address the complexity of biofilm formation. This study hypothesised that different $S$. aureus protein ( $\mathrm{Spa}$ ) gene sequencing, multilocus sequence typing (MLST), and Staphylococcal cassette chromosome mec (SCCmec) typing are among the factors that affect the ability of the bacteria to form biofilms. The study carries out phenotypic and genotypic investigation to test this hypothesis and to discover factors that affect the differences in adherence and biofilm production rate and characteristics. Differences occurring due to clonal variation would indicate a need for accurate clonal identification for effective biofilm management upon infection.

\section{Materials and Methods}

2.1. Bacterial Isolates. The study used a total of 30 different clinical MRSA isolates containing 13 different Spa types, the identity of which was confirmed from a previous epidemiology study conducted by Ghaznavi-Rad et al. [5], and received in the form of stock culture from the Medical Microbiology Laboratory at the University Putra Malaysia for use in this study. The Kuala Lumpur General Hospital provided all of the isolates that were obtained from different systemic infection sites. The isolates were characterised as different clones using SCCmec, Spa, and MLST. The reference strains used were the positive biofilm producer ATCC 35556, negative biofilm producer ATCC 12228, and ATCC700698 for MRSA.

\subsection{Biofilm Assay}

2.2.1. Congo Red Agar. The study used Congo red agar (CRA) that had been modified as described by Mariana et al. [6] to detect slime producing various isolates. The agar consisted of $10 \mathrm{~g}$ of glucose (Baker.UK) with $0.4 \mathrm{~g}$ of Congo red (Sigma) in one litre of Blood Base Agar-2 (BAB-2) [Oxoid, Basingstoke, Hampshire, England]. The isolates were incubated at $37^{\circ} \mathrm{C}$ for $48 \mathrm{~h}$ under aerobic conditions. The experiment was repeated three times. The colonies produced were labelled as very black, black, and weak black. These black colonies were considered to be normal slime producing, while red colonies were classified as non-slimeproducing bacteria [7].

2.2.2. Quantitative Microtiter Plate Method. The microtiter plate method used by Stepanović et al. [8] with minor modification was used in this study to determine the quantities of the biofilm. Briefly, all the MRSA isolates were grown for $48 \mathrm{~h}$ under aerobic conditions in 6-well polystyrene tissue culture plates supplemented with trypticase soy broth (TSB) containing $1 \%$ glucose at $37^{\circ} \mathrm{C}$. The plates were then washed ten times in deionised water. The remaining attached bacteria were fixed with $2 \mathrm{~mL}$ of absolute methanol per well for $20 \mathrm{~min}$ before the plates were emptied and left to dry overnight. The biofilm was stained with $1 \mathrm{~mL}$ of $0.1 \%$ safranine for $15 \mathrm{~min}$. Excess stain was rinsed off, and the plates were washed three times with distilled water. After the plates were air dried overnight, the dye that was bound to the adherent cells was dissolved with $95 \%$ ethanol per well, and absorbance was measured at $490 \mathrm{~nm}\left(\mathrm{~A}_{490}\right)$. The experiment was repeated three times. The absorbance $\left(\mathrm{A}_{490}\right)$ of wells containing sterile TSB was used as the negative control. An $\mathrm{A}_{490}$ value of 0.5 was selected as the cutoff point to differentiate as positive for biofilm formation $(\mathrm{ODc}=$ average $\mathrm{OD}$ of negative control $+3 \mathrm{x}$ SD of negative control). Biofilm formation for each isolate was analysed and categorised based on the absorbance of the safranine-stained biofilm. Isolates were considered as highly adherent ++++ $\left(\mathrm{A}_{490}>3.0\right)$, strongly adherent $+++\left(\mathrm{A}_{490}>2.0\right)$, moderately adherent $++\left(\mathrm{A}_{490}>1.0-2.0\right)$, weakly adherent $+\left(\mathrm{A}_{490}>\right.$ $0.5-1.0)$, and negatively adherent $-\left(\mathrm{A}_{490} \leq 0.5\right)$ on the basis of their absorbance property. In this study, the isolates with weak to highly strong adherence $\left(\mathrm{A}_{490}>0.5\right)$ were labelled as biofilm producers.

2.2.3. Light Microscope. Biofilm production was also assessed by a Nikon Eclipse light microscope (4000x magnification using a VM C-4X Lens, Nikon, Japan) and colony counter (Stuart Scientific, UK). The bacteria were grown in six-well tissue culture plates on glass coverslips in TSB containing 1\% glucose at $37^{\circ} \mathrm{C}$ for $48 \mathrm{~h}$. Bacteria growing as aggregates on the glass coverslip surfaces were visualised and photographed by a Nikon light microscope equipped with a digital camera.

2.2.4. Scanning Electron Microscopy (SEM). Biofilm formation was further confirmed by electron microscopy to distinguish the biofilm producers from the nonproducers. Two methods were used in the preparation of samples for scanning electron microscopy: one, a method described by Johan et al. [9] to determine the production of extracellular matrix, and two, a conventional method described by Ganderton et al. [10] to determine the network layers of a biofilm. In the first experiment, bacteria were grown on glass coverslips placed in six wells of tissue culture plates at $37^{\circ} \mathrm{C}$ for $48 \mathrm{~h}$ and then fixed with $70 \%$ methanol for $20 \mathrm{~min}$. The samples were subjected to air drying overnight, after which the samples were mounted using a double-sided tape and then gold coated using a gold sputtering unit (spray coating, Bal-Tec SCD 005), and later examined using SEM. In the second experiment, bacteria were grown on the surface of glass coverslips, fixed in $4 \%$ buffered glutaraldehyde for $5 \mathrm{~h}$, and rinsed three times with $0.1 \mathrm{M}$ sodium cacodylate buffer. The sample was then fixed in $0.1 \mathrm{M}$ osmium tetroxide for $1 \mathrm{~h}$ at $4^{\circ} \mathrm{C}$, dehydrated with $35-100 \%$ ethanol in double distilled water, and dried in a critical point dryer, after which the samples were gold coated as above.

2.3. Genotyping Methods. Total chromosomal DNA from the different MRSA isolates were extracted using a DNeasy 
kit (Qiagen Inc.) according to the manufacturer's instructions. The sequences of icaADBC, bbp, clfA, clfB, cna, $e b p S$, eno, $f n b A, f n b B$, and $f i b$ genes were taken from the National Centre for Biotechnology Information (http://www .ncbi.nlm.nih.gov/) GenBank sequence database with accession numbers AF086783, Y18653, Z18852, AJ224764, M81736, U48826, AF065394, X95848, X62992, and X72014, respectively, and were commercially synthesised by EUROGENTEC AIT/SINGAPORE139552. The sequences of the primers and the thermocycling conditions for each primer used in this study were previously described by Cramton et al. [11], Kiem et al. [12], Vancraeynest et al. [13], Cucarella et al. [14], Montanaro et al. [15] and Tristan et al. [16]. The master reaction mixtures for each primer were in $25 \mu \mathrm{L}$ volumes, and the amplified products were analysed by agarose gel electrophoresis using 1.2\% agarose concentration in Trisborate-EDTA buffer and compared to a $100 \mathrm{bp}$ molecular weight marker. The gel was viewed under UV light in an Alphalmager ${ }^{\mathrm{R}}$ Imaging System, and the image was evaluated for biofilm production in relation to the presence or absence of biofilm and adhesion genes in the MRSA isolates.

2.4. Statistical Analysis. The difference in the mean values of quantified biofilm adherence between two groups was tested for significance using chi-square or fisher exact tests and the difference between more than two groups was tested using one-way ANOVA. $P$ values of $<0.05$ were considered significant.

\section{Results}

\subsection{Biofilm Assay}

3.1.1. Congo Red Agar. As shown in Table 1, 30 different isolates of MRSA were determined to be slime producers to varying degrees, including 7 isolates $(23.33 \%)$ that were very black, $20(66.66 \%)$ that were black, $1(3.33 \%)$ that was weak black, and $2(6.66 \%)$ that were red. The S. aureus ATCC 35556 slime-producing strain produced typical black colonies after $48 \mathrm{~h}$ of incubation. The non-slime-producing S. epidermidis ATCC 12228 produced pink colonies.

3.1.2. Quantitative Microtiter Plate Method. Figures 1 and 2 show that $100 \%$ of the different MRSA isolates produced biofilm that was adherent at varied levels: $2(6.66 \%)$ isolates were weakly adherent with $\mathrm{OD}_{490}$ values of $>0.5-1.0,8$ isolates (26.66) were moderately adherent with $\mathrm{OD}_{490}$ values of $>1.0-2.0,11$ isolates (36.66) were strongly adherent with $\mathrm{OD}_{490}$ values of $>2.0$, and 9 isolates were highly adherent with $\mathrm{OD}_{490}$ values of $>3.0$. The ATCC 35556 strain was strongly adherent with an $\mathrm{OD}_{490}$ value $>2.0$, while the ATCC 12228 strain was negatively adherent $\left(\mathrm{OD}_{490}<0.5\right)$.

3.1.3. Light and Scanning Electron Microscopy. Figure 3 shows direct visualisation of similar autoaggregates in liquid culture growth of highly, strongly, moderately, and weakly adherent isolates on the glass coverslip surfaces after $48 \mathrm{~h}$ incubation using a Nikon Eclipse light microscope and colony counter lens. These autoaggregates can be compared to the nonaggregate produced by the $S$. epidermidis ATCC 12228 reference strain, shown in Figure 4.

Using scanning electron microscopy, we observed that the isolates that were weakly to highly adherent formed thick extracellular products surrounding the cells aggregated on the coverslip surface when the bacteria were fixed with $70 \%$ methanol (Figure 5(a)), whereas the nonadherent ATCC 12228 did not produce extracellular products (Figure 5(b)). Using the conventional method of SEM, clones characterised by weak to high adherence formed varying degrees of biofilm production. Clones characterised by high and strong adherence produced large thick layers (Figure 6(a)), while the clones characterised by moderate and weak adherence formed less of a network among themselves (Figure 6(b)). The non-adherent ATCC 12228 reference strain showed an absence of biofilm network layers (Figure 6(c)).

3.2. Detection of Biofilm and Adhesion Loci by PCR. As presented in Table 1, we found that the clones that were positive for the icaA gene were also positive for the $i c a \mathrm{D}, i c a \mathrm{~B}, i c a \mathrm{C}$, $f n b A, e n o, e b p s, c l f A$, and $c l f B$ genes, while the prevalence of $f i b, c n a, f n b B$, and $b b p$ in MRSA clones were 90, 93.33, 53.33 and $10 \%$, respectively.

\section{Discussion}

This study attempts to investigate biofilm formation by different isolates of MRSA with a number of different methods to determine the various attachment and biofilm phenotypes. Table 1 shows the variable slime and adherentforming abilities of 30 different of MRSA isolates tested. Some isolates belonging to the same type of Spa, such as t037, were found to form a highly adherent biofilm using the microtiter plate method but had heterogeneous results on the Congo red agar assay. However, some isolates belonging to different Spa types, such as $t 4184$ and t4213, were negative for producing slime on the Congo red agar but were observed to have weak and strong biofilm production by the microtiter plate method, respectively. Thus, slime phenotypic diversity on Congo red agar for those isolates that belong to the same or different Spa-MLST-SCCmec types may result from heterogeneity in genetic backgrounds or because of differences in interpretation of colonies based on their colour and not on their morphology. However, isolates that belong to the same Spa, MLST, and SCCmec types showed a similarity in adherence capacity by the microtiter plate method (see Figure 2), and significant differences were found between different Spa types. For example, the 1932 clone that belongs to the ST-239-CC8-IIIA type was weakly adherent, while the 037 clone belonging to the same ST-239- CC8-IIIA type was highly adherent, four times more adherent than the weakly adherent clone $(P<0.05)$. Thus, variation is due to the different Spa types and not due to the MLST or SCCme typing. The ability to produce biofilms is not dependent on SCCmec; rather, it is dependent on the lineage. This is in agreement with similar findings by Croes et al. [17] reported differences in adherence quantities among $S$. aureus isolates associated with the genetic background of protein $\mathrm{A}(\mathrm{Spa})$. 


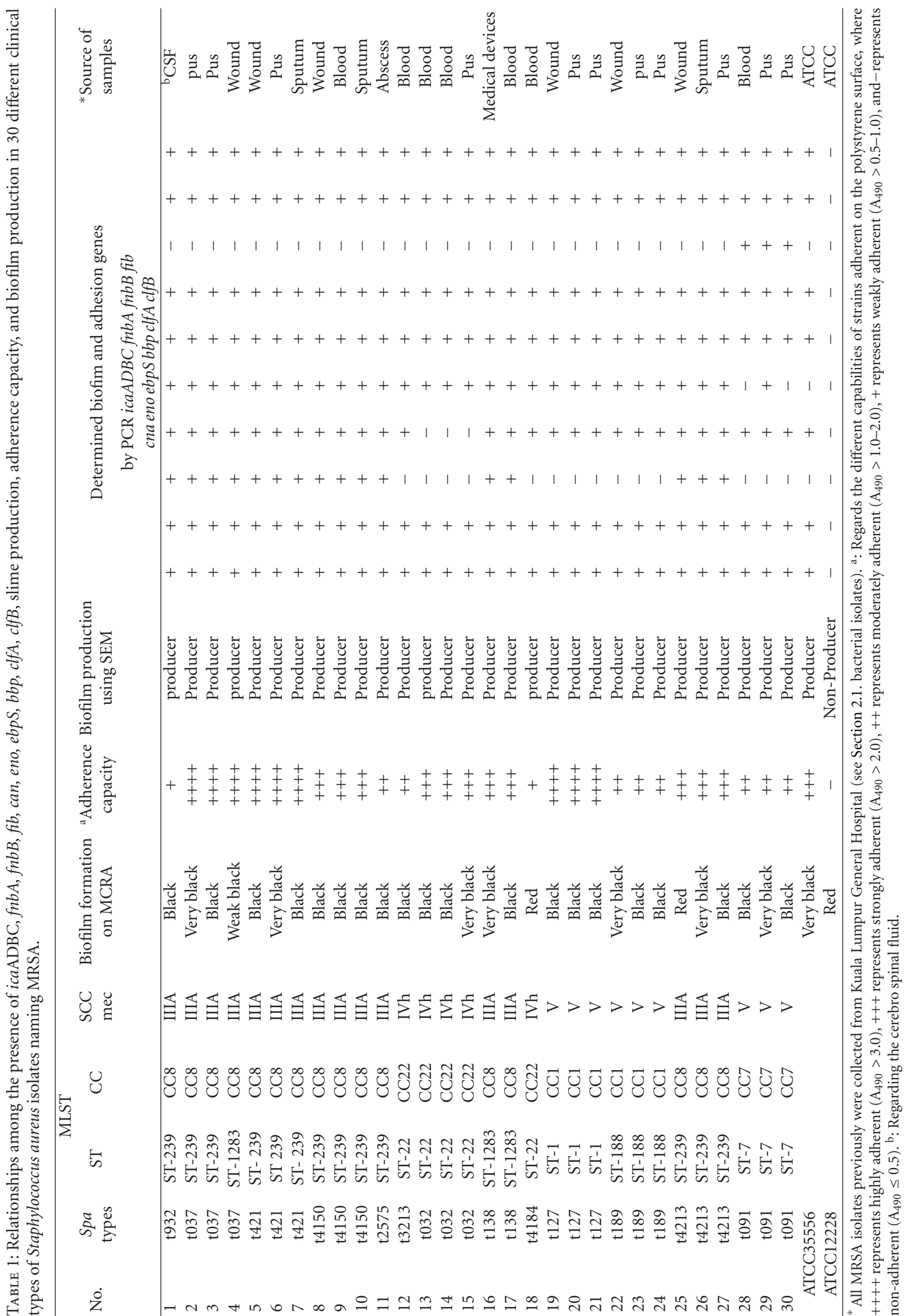




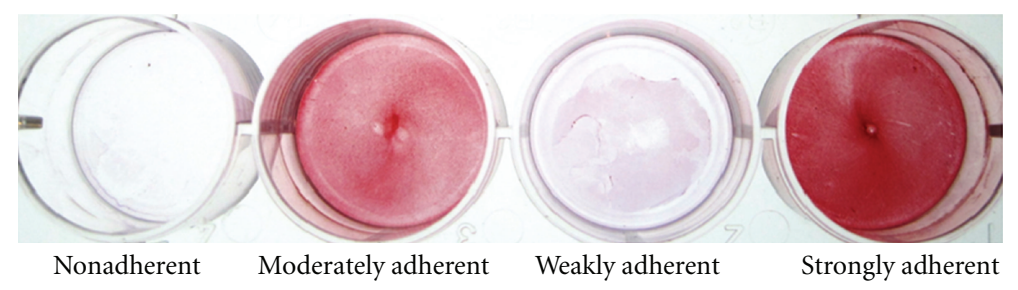

FIGURE 1: Total biofilm formation of different clinical MRSA isolates. The bacteria were grown in 6-well tissue culture plates containing TSB supplemented with $1 \%$ glucose. The cells that adhered to the plate surface after washing were visualised by staining with safranin. The clones were considered as highly, strongly, moderately, weakly, and nonadherent based upon their absorbance as measured by a spectrophotometer.

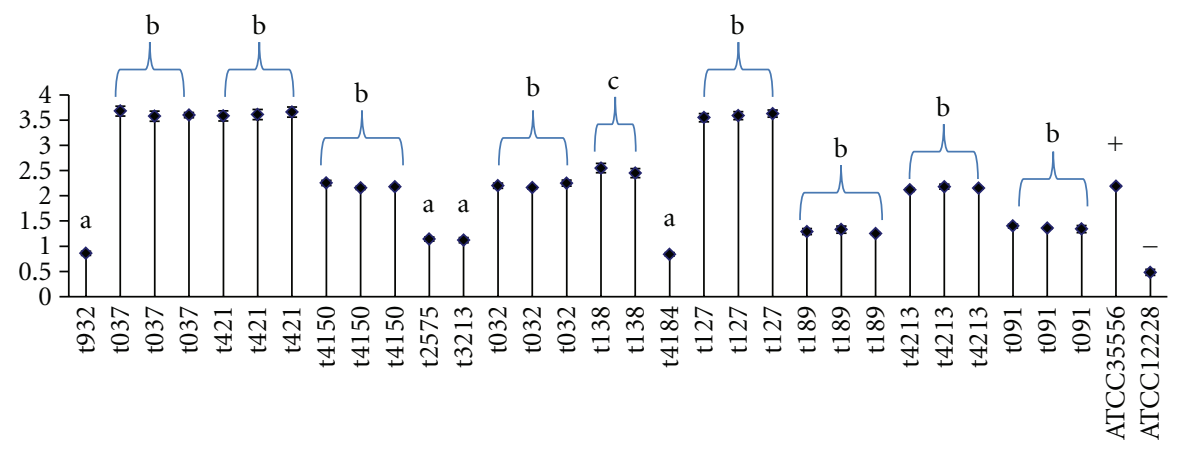

- OD490

Figure 2: A chart displaying each spa type measures the quantity of biofilm using a microtiter plate assay for all isolates of this spa type represented as dots. Data represent the mean of triplicate determinations of the increase in bacterial adhesion. An OD value of 0.5 was selected as the cut-off point; any OD value above the cutoff OD was considered positive for biofilm formation. The isolates of one clone have similar quantitative biofilm-forming properties. The isolates of different clones have different quantitative biofilm forming properties. (a) Indicates to the single frequently isolates belonging to different spa typing. (b) Indicates to the three similar isolates of one clone. (c) Indicates to the two similar isolates of one clone. +: Indicates to the positive biofilm reference strain. - : Indicates to the negative biofilm reference strain.
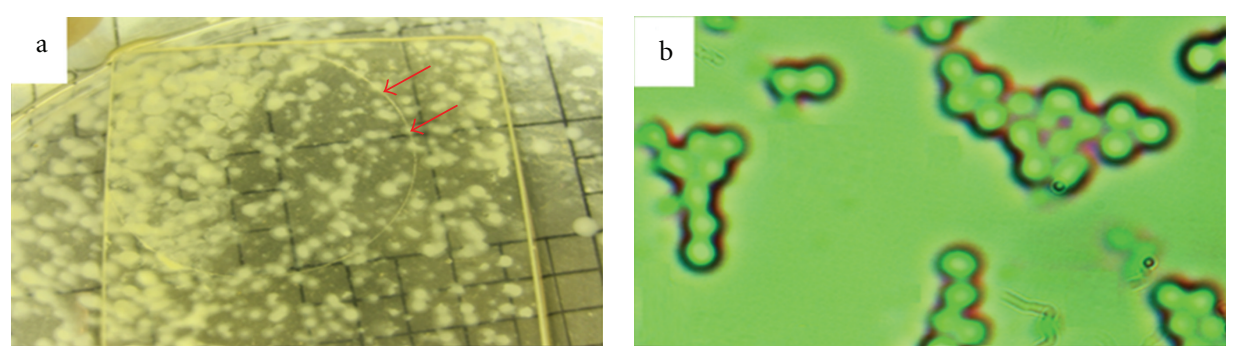

FIGURE 3: Direct visualisation of bacterial autoaggregation in liquid culture media of strongly, moderately, and weakly adherent isolates on a glass coverslip surface after a $48 \mathrm{~h}$ incubation using a colony counter lens (a) and the Nikon Eclipse light microscope (b).
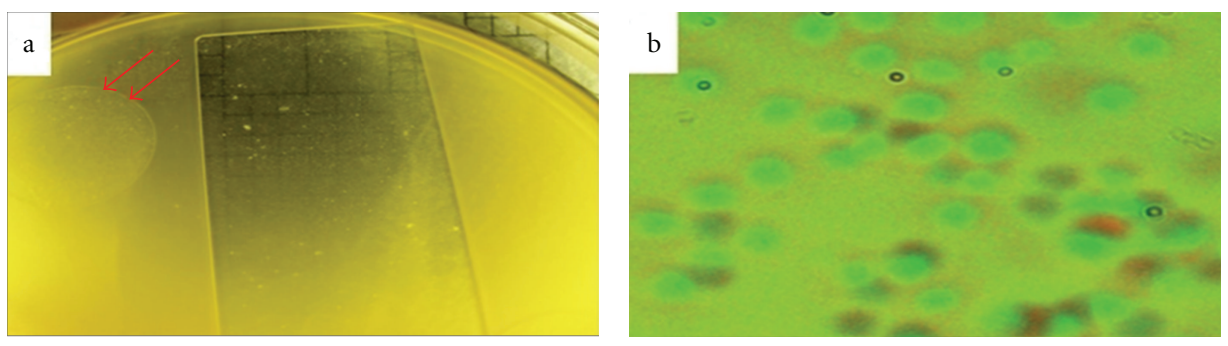

FIGURE 4: Direct visualisation of bacterial auto-aggregation in liquid culture media of the non-adherent Staphylococcus epidermidis ATCC 12228 reference strain on a glass coverslip surface after a $48 \mathrm{~h}$ incubation using a colony counter lens (a) and the Nikon Eclipse light microscope (b). 

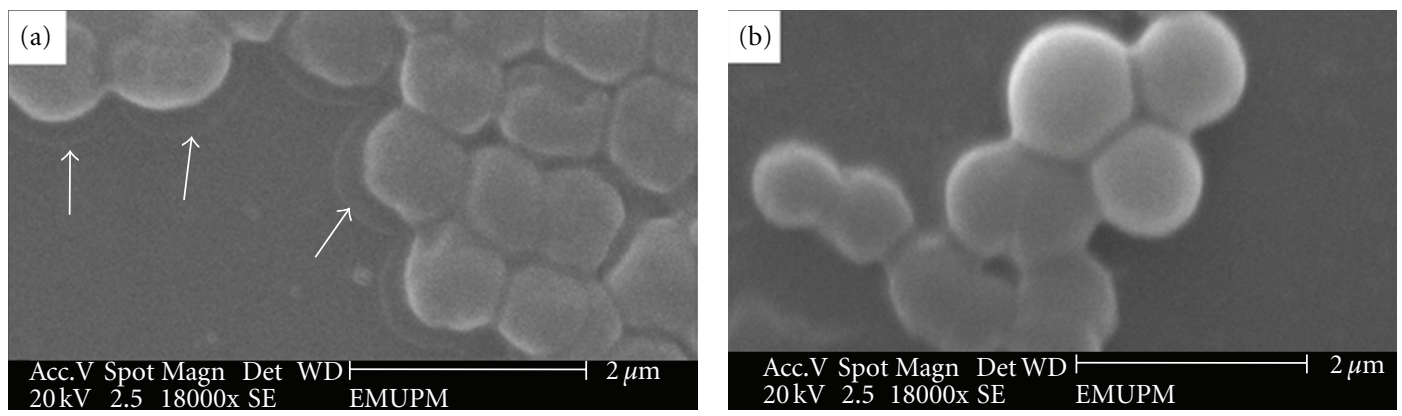

FIGURE 5: A scanning electron micrograph illustrating the appearance of thick extracellular products in strongly, moderately, and weakly adherent isolates (a) and the absence of those products in the non-adherent Staphylococcus epidermidis ATCC 12228 reference strain (b).
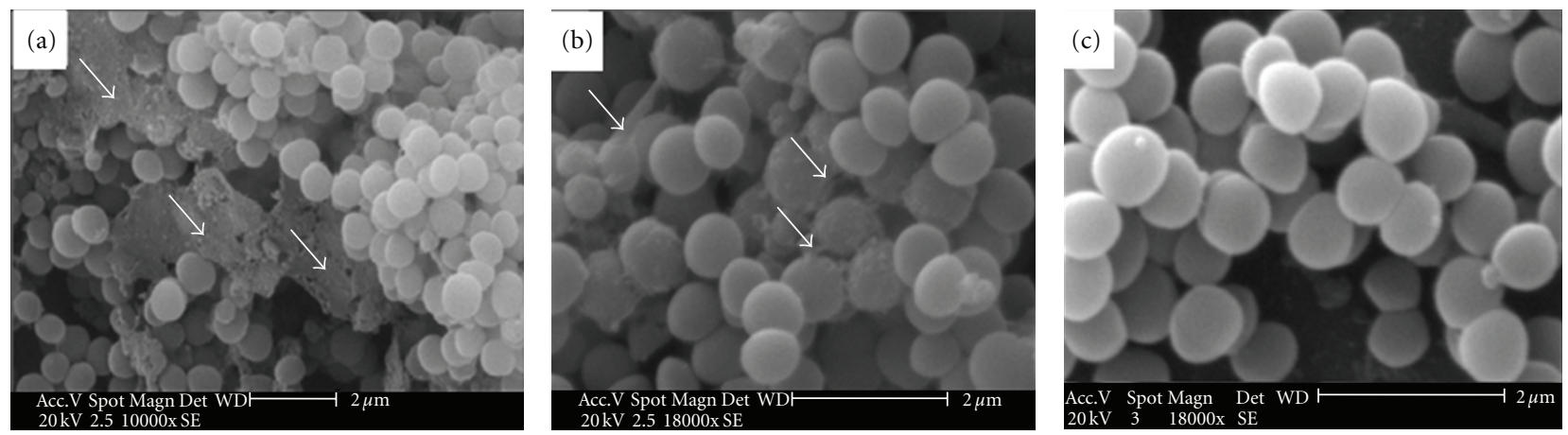

FIGURE 6: A scanning electron micrograph revealing variable degrees of heavy biofilm layer production. Highly and strongly adherent isolates produced a large thick layer (a), and moderately and weakly adherent isolates formed a lower degree of a close network among themselves (b), while negative biofilm producers showed an absence of network layers (c).

It is likely that the cell-to-cell aggregation and adhesion observed by others was done with a method that is more efficient for the investigation of biofilm production. Direct visualisation using light microscopy at high magnification revealed that biofilms were formed at the beginning of bacterial adhesion, suggesting that the formation of aggregates and adhesion to biomaterial surfaces is a critical event during early biofilm formation. It is worth noting that in this method, it is difficult to differentiate between aggregations of weakly, and highly adherent clones; the weakly, moderately, strongly and highly adherent clones formed similar aggregates on the glass surfaces (Figure 3), while the negative biofilm ATCC 12228 reference strain did not form aggregates, as shown in Figure 4. However, to confirm the presence of an extracellular polysaccharide and glycoprotein network layer, scanning electron microscopy was used. In the conventional preparatory technique, using dehydration through a graded ethanol and critical point drying, the extracellular matrix biofilm was washed off through exposure to the numerous fluids used in this process (Johan et al. [9]). A modified technique was developed whereby the specimens were air dried directly after fixation with $70 \%$ methanol and prior to coating. Reproducible and consistent results were obtained by following this procedure, as can be seen in the 30 isolates that were visibly and clearly within a extracellular polysaccharide network (Figure 5(a)), while the ATCC 12228 reference strain did not produce extracellular polysaccharides (Figure $5(\mathrm{~b})$ ). This modified method has given reliable results for determining extracellular polysaccharides but is not suitable for determining the glycoprotein biofilm network layer because of the ambiguities caused by dehydration using ethanol. The conventional technique was therefore used to determine the glycoprotein network layer. The highly and strongly adherent isolates formed a rich glycoprotein network layer (Figure 6(a)), while a poor glycoprotein network layer was formed only in isolates that were weakly and moderately adherent, as demonstrated in Figure 6(b). Photographs of the non-adherent ATCC reference strain are shown in Figure 6(c). Genotypic studies using PCR showed that all 30 isolates of MRSA tested were $100 \%$ positive for the presence of the icaADBC, fnbA, eno, $e b p s, \operatorname{clf} A$, and $c l f B$ genes. In this study, we found very similar results obtained using SEM and the microtiter plate assay with regard to the presence of the icaADBC and some of adhesion genes as a determining factor for the level of biofilm production by different clones.

\section{Conclusions}

The findings of this study using qualitative and quantitative methods have shown that genotypically different isolates of MRSA have different capabilities to produce biofilms. This diversity in biofilm characteristics is due to the different Spa type, not the MLST or SCCmec type. Therefore, in general, 
a number of different phenotypic and genotypic methods are necessary for the precise identification of clones for effective biofilm management upon infection.

\section{Acknowledgment}

The authors take this opportunity to thank the Department of Medical Microbiology and Parasitology, Putra University, Malaysia for kindly providing all of the MRSA isolates.

\section{References}

[1] P. Stoodley, K. Sauer, D. G. Davies, and J. W. Costerton, "Biofilms as complex differentiated communities," Annual Review of Microbiology, vol. 56, pp. 187-209, 2002.

[2] H. M. Probert and G. R. Gibson, "Bacterial biofilms in the human gastrointestinal tract," Current Issues in Intestinal Microbiology, vol. 3, no. 2, pp. 23-27, 2002.

[3] C. Heilmann, C. Gerke, F. Perdreau-Remington, and F. Götz, "Characterization of Tn917 insertion mutants of Staphylococcus epidermidis affected in biofilm formation," Infection and Immunity, vol. 64, no. 1, pp. 277-282, 1996.

[4] K. Chaieb, K. Mahdouani, and A. Bakhrouf, "Detection of icaA and icaD loci by polymerase chain reaction and biofilm formation by Staphylococcus epidermidis isolated from dialysate and needles in a dialysis unit," Journal of Hospital Infection, vol. 61, no. 3, pp. 225-230, 2005.

[5] E. Ghaznavi-Rad, M. N. Shamsudin, Z. Sekawi et al., "Predominance and emergence of clones of hospital-acquired methicillin-resistant Staphylococcus aureus in Malaysia," Journal of Clinical Microbiology, vol. 48, no. 3, pp. 867-872, 2010.

[6] N. S. Mariana, S. A. Salman, V. Neela, and S. Zamberi, "Evaluation of modified Congo red agar for detection of biofilm produced by clinical isolates of methicillin resistance Staphylococcus aureus," African Journal of Microbiology Research, vol. 3, no. 6, pp. 330-338, 2009.

[7] C. R. Arciola, D. Campoccia, L. Baldassarri et al., "Detection of biofilm formation in Staphylococcus epidermidis from implant infections. Comparison of a PCR-method that recognizes the presence of ica genes with two classic phenotypic methods," Journal of Biomedical Materials Research A, vol. 76, no. 2, pp. 425-430, 2006.

[8] S. Stepanović, D. Vuković, V. Hola et al., "Quantification of biofilm in microtiter plates: overview of testing conditions and practical recommendations for assessment of biofilm production by Staphylococci," APMIS, vol. 115, no. 8, pp. 891$899,2007$.

[9] V. Johan, H. Martin, H. Danie, and M. Johan, "Antimicrobial coating agents: can biofilm formation on a breast implant be prevented?" Journal of Plastic, Reconstructive and Aesthetic Surgery, vol. 62, no. 5, pp. 610-617, 2009.

[10] L. Ganderton, J. Chawla, C. Winters, J. Wimpenny, and D. Stickler, "Scanning electron microscopy of bacterial biofilms on indwelling bladder catheters," European Journal of Clinical Microbiology and Infectious Diseases, vol. 11, no. 9, pp. 789796, 1992.

[11] S. E. Cramton, C. Gerke, N. F. Schnell, W. W. Nichols, and F. Götz, "The intercellular adhesion (ica) locus is present in Staphylococcus aureus and is required for biofilm formation," Infection and Immunity, vol. 67, no. 10, pp. 5427-5433, 1999.

[12] S. Kiem, W. S. Oh, K. R. Peck et al., "Phase variation of biofilm formation in Staphylococcus aureus by IS256 insertion and its impact on the capacity adhering to polyurethane surface," Journal of Korean Medical Science, vol. 19, no. 6, pp. 779-782, 2004.

[13] D. Vancraeynest, K. Hermans, and F. Haesebrouck, "Genotypic and phenotypic screening of high and low virulence Staphylococcus aureus isolates from rabbits for biofilm formation and MSCRAMMs," Veterinary Microbiology, vol. 103, no. 3-4, pp. 241-247, 2004.

[14] C. Cucarella, C. Solano, J. Valle, B. Amorena, I. Lasa, and J. R. Penadés, "Bap, a Staphylococcus aureus surface protein involved in biofilm formation," Journal of Bacteriology, vol. 183, no. 9, pp. 2888-2896, 2001.

[15] L. Montanaro, C. Renata Arciola, L. Baldassarri, and E. Borsetti, "Presence and expression of collagen adhesin gene (cna) and slime production in Staphylococcus aureus strains from orthopaedic prosthesis infections," Biomaterials, vol. 20, no. 20, pp. 1945-1949, 1999.

[16] A. Tristan, L. Ying, M. Bes, J. Etienne, F. Vandenesch, and G. Lina, "Use of multiplex PCR to identify Staphylococcus aureus adhesins involved in human hematogenous infections," Journal of Clinical Microbiology, vol. 41, no. 9, pp. 4465-4467, 2003.

[17] S. Croes, R. H. Deurenberg, M. L. L. Boumans, P. S. Beisser, C. Neef, and E. E. Stobberingh, "Staphylococcus aureus biofilm formation at the physiologic glucose concentration depends on the S. aureus lineage," BMC Microbiology, vol. 9, article 229, 2009. 

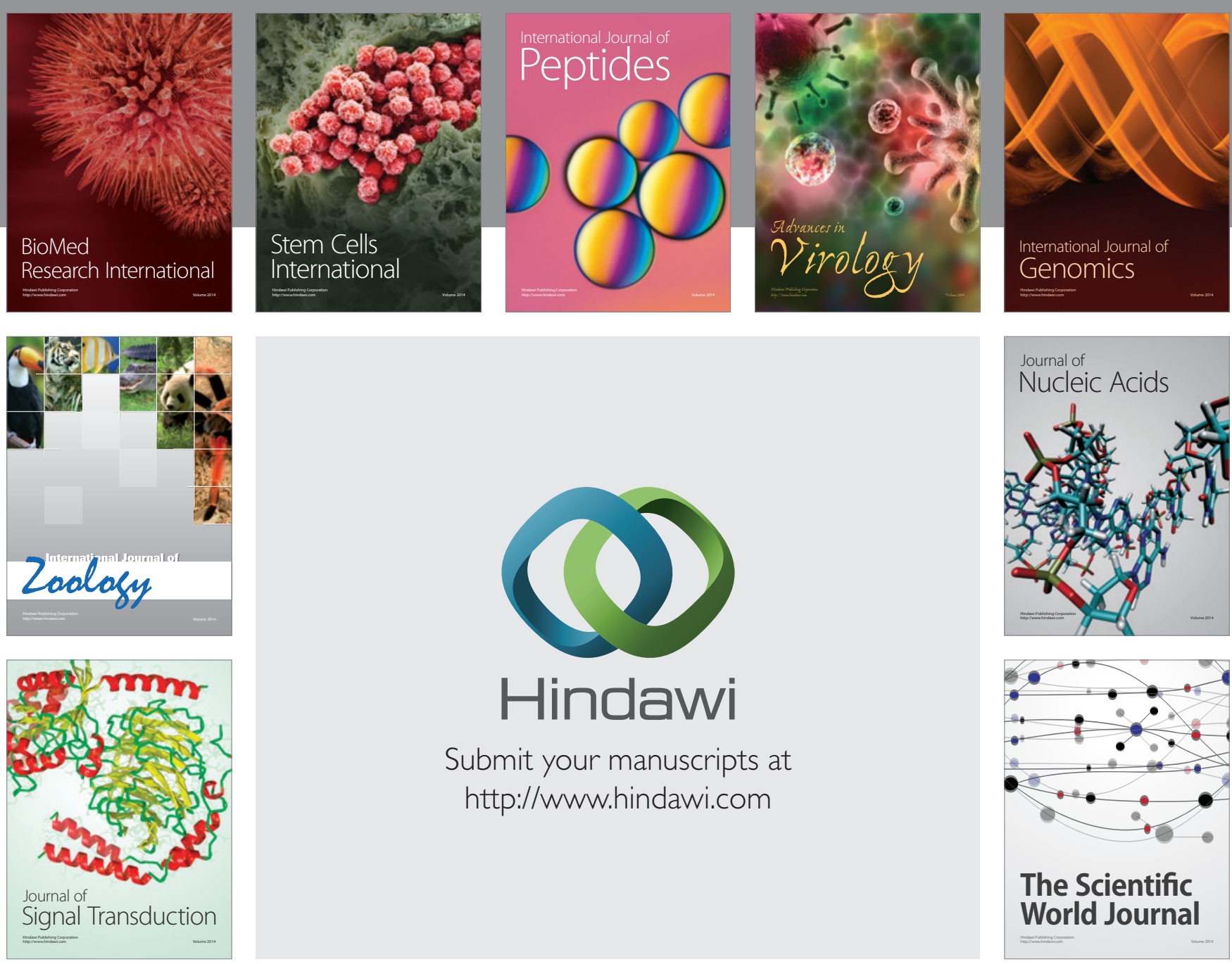

Submit your manuscripts at

http://www.hindawi.com
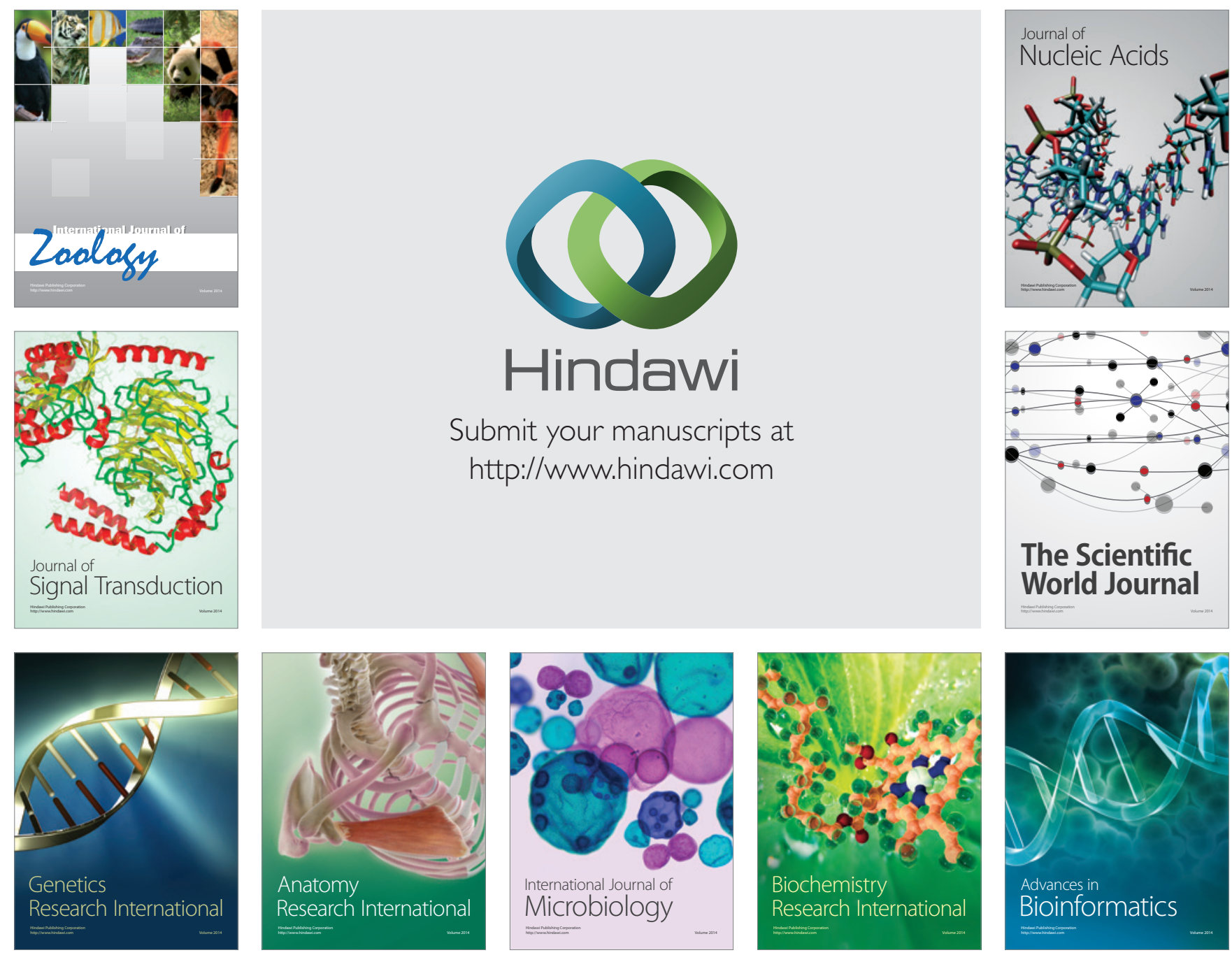

The Scientific World Journal
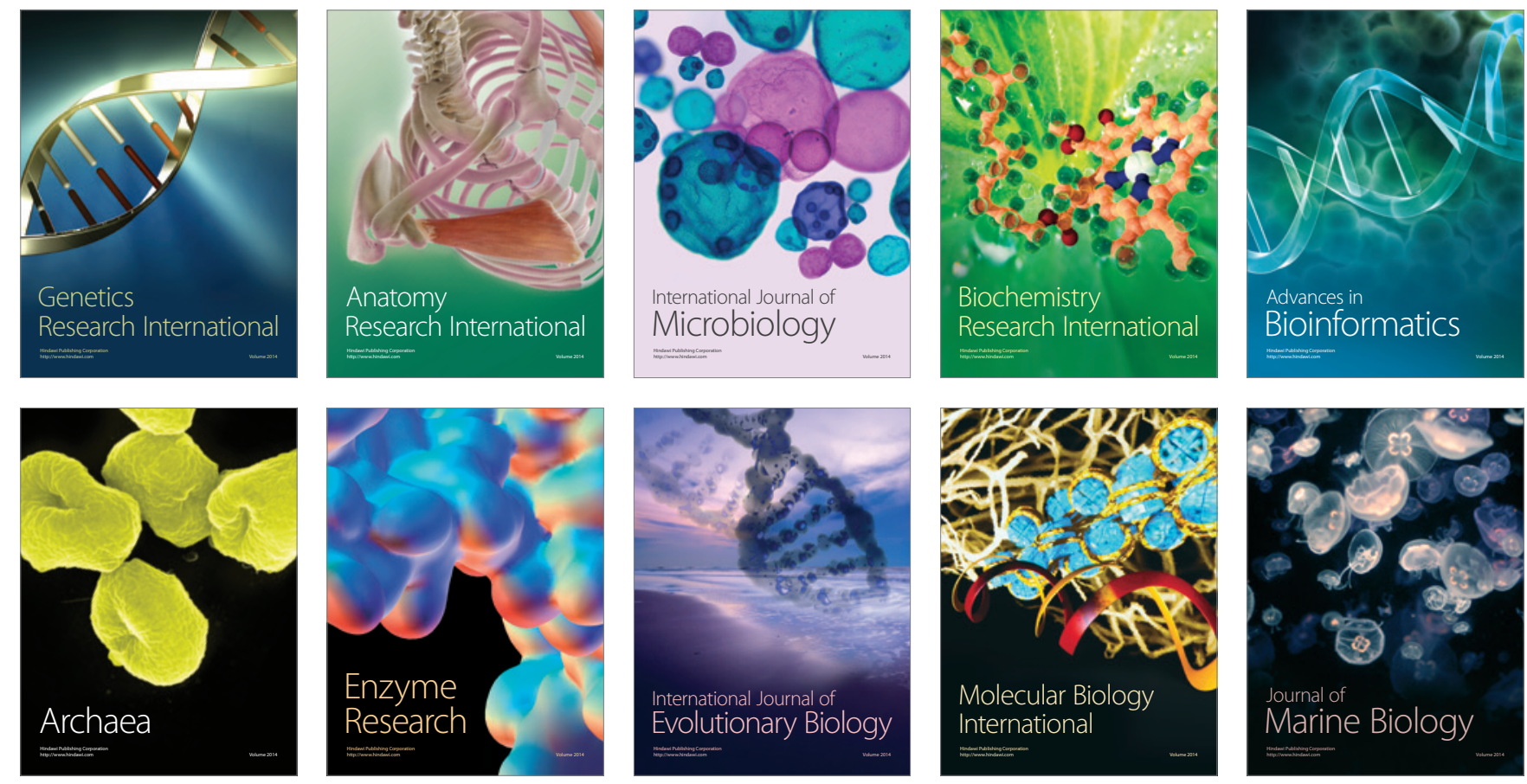